\title{
Precision QCD Measurements in DIS at HERA
}

\author{
DANIEL BRITZGER \\ on behalf of the $\mathrm{H} 1$ and ZEUS Collaborations \\ Deutsches Elektronen-Synchrotron, Notkestr. 85, 22607 Hamburg, Germany \\ daniel.britzger@desy.de
}

Received (Day Month Year)

Revised (Day Month Year)

\begin{abstract}
New and recent results on QCD measurements from the H1 and ZEUS experiments at the HERA ep collider are reviewed. The final results on the combined deep-inelastic neutral and charged current cross sections are presented and their role in the extractions of parton distribution functions (PDFs) is studied. The PDF fits give insight into the compatibility of QCD evolution and heavy flavor schemes with the data as a function of kinematic variables such as the scale $Q^{2}$. Measurements of jet production cross sections in $e p$ collisions provide direct proves of $\mathrm{QCD}$ and extractions of the strong coupling constants are performed. Charm and beauty cross section measurements are used for the determination of the heavy quark masses. Their role in PDF fits is investigated. In the regime of diffractive DIS and photoproduction, dijet and prompt photon production cross sections provide insights into the process of factorization and the nature of the diffractive exchange.
\end{abstract}

Keywords: QCD; HERA; Deep-inelastic scattering; jet production.

PACS Nos.: include PACS Nos.

\section{Introduction}

Deep-inelastic scattering (DIS) data of electron ${ }^{\mathrm{a}}$-proton interactions provide an excellent tool for studies of the phenomenology and parameters of Quantum Chromodynamics (QCD). Of particular importance are the high-precision inclusive measurements of neutral and charged current processes. These give access to proton parton density functions (PDFs) by means of QCD fits based on the DGLAP evolution equations. On the other hand, measurements of selected QCD induced final states provide a more direct access to certain QCD properties. Examples are the production of jets or heavy flavours.

Data in deep-inelastic $e^{ \pm} p$ scattering have been recorded in the years $1992-$ 2007 by the two multi-purpose experiments $\mathrm{H} 1$ and ZEUS at different center-ofmass energies with electron beam energies of $E_{e} \simeq 27 \mathrm{GeV}$. The data taking periods

aThe term 'electron' also includes positrons, since HERA was operated with both leptons. 
were divded into two running periods, HERA-I in which about $0.1 \mathrm{fb}^{-1}$ and HERAII in which almost $0.4 \mathrm{fb}^{-1}$ of integrated luminosity was recorded per experiment. Neutral (NC) and charged current (CC) interactions are mediated by virtual $Z / \gamma^{*}$ or $W$, respectively, and the kinematical reach in four-momentum transfer of the exchanged boson $\left(\sqrt{Q^{2}}\right)$ ranges from $0.045 \leq Q^{2} \leq 50000 \mathrm{GeV}^{2}$ and in the scaling variable $x_{\mathrm{Bj}}$ down to $6 \cdot 10^{-7}$ as shown in fig. 1 . Several years after data taking, both

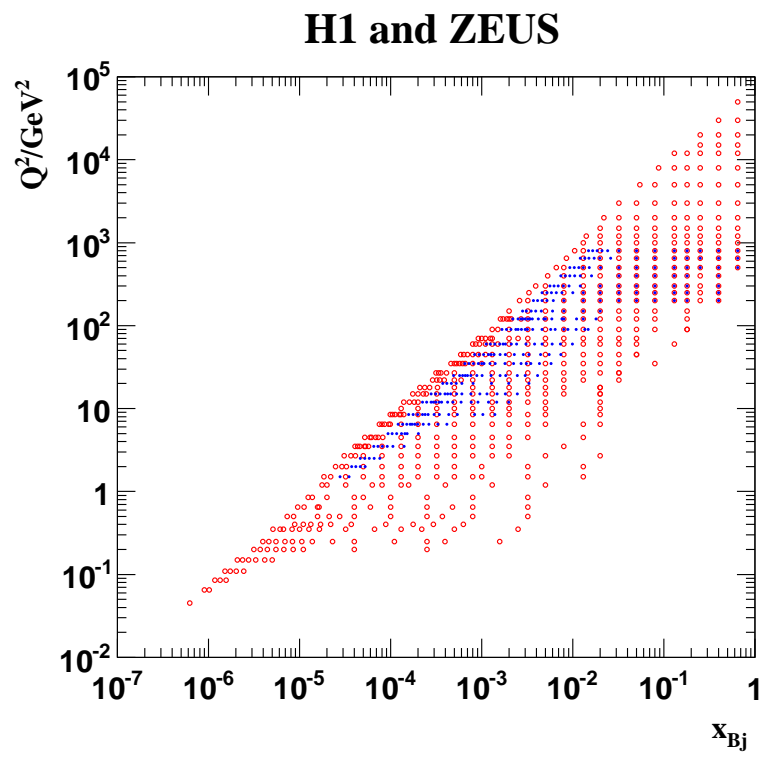

Fig. 1. The points of the two grids used for the combination of the inclusive DIS cross sections illustrating the huge kinematic range of the HERA $e p$ data in DIS. Circles were used for data with $\sqrt{s}=318 \mathrm{GeV}$; thin dots were used for data with $\sqrt{s}=251 \mathrm{GeV}$ or $\sqrt{s}=225 \mathrm{GeV}$.

experiments have successively refined their analysis techniques and are presenting results with their final precision. Further improvements of the precision of the cross sections is often achieved by a combination of results from different data taking periods and in particular from both of the experiments.

The measurements of the inclusive deep-inelastic NC and CC cross sections play an outstandig role, since these are major ingredients to the extraction of parton density functions (sec. 2). On the other side the measurements of the final state, like jet production (sec. 3) or heavy flavor production cross sections (sec. 4), provide direct probes of QCD and give access to the fundamental parameters of QCD such as the strong coupling constant $\alpha_{s}\left(M_{Z}\right)$ or heavy quark masses. 


\section{Inclusive deep-inelastic scattering}

The reduced cross section of neutral current $e^{ \pm} p$ deep-inelastic scattering, $e^{ \pm} p \rightarrow$ $e^{ \pm}+X$, can be generically written in terms of structure functions

$$
\sigma_{\mathrm{r}, \mathrm{NC}}^{ \pm}=\tilde{F}_{2}^{ \pm} \mp \frac{1-(1-y)^{2}}{1+(1-y)^{2}} x \tilde{F}_{3}^{ \pm}-\frac{y^{2}}{1+(1-y)^{2}} \tilde{F}_{L}^{ \pm},
$$

with $y$ being the inelasticity. The generalized structure functions $\tilde{F}_{2}^{ \pm}, x \tilde{F}_{3}^{ \pm}$and $\tilde{F}_{L}^{ \pm}$can be written as linear combinations of the proton structure functions $F_{2}$, $F_{2,3}^{\gamma Z}$, and $F_{2,3}^{Z}$ which contain the informations on the QCD parton dynamics and are associated to pure photon exchange, to the photon- $Z$ interference terms and to pure $Z$-exchange. The longitudinal structure function $F_{L}$ contributes only at high $y$, while the $F_{3}$ structure function contributes only at high values of $Q^{2}$. In the quarkparton model, the structure functions are linear combinations of the quark and anti-quark momentum distributions $x q\left(x, Q^{2}\right)$ and $x \bar{q}\left(x, Q^{2}\right)$. The charged current cross sections may be similarly written in terms of structure functions, $W_{2}^{ \pm}, x W_{3}^{ \pm}$, and $W_{L}$ for charged current scattering.

\subsection{Combined inclusive neutral and charged current cross sections}

The entire set of $\mathrm{H} 1$ and ZEUS inclusive $\mathrm{NC}$ and CC cross section measurements are input to a combination procedure to obtain a consistent set of cross section data $^{1}$. The combination was performed using the HERAAverager package ${ }^{2}$, which determines the combined data points with their statistical and experimental uncertainties using a $\chi^{2}$ procedure. In total 2927 input data points are combined to 1307 points. If needed, small factors to correct to common phase space points are derived with the help of the finally extracted PDFs. The combination exhibits a value of $\chi^{2}=1687$ for 1620 degrees of freedom, and thus demonstrates the great consistency of the data over the different running periods and the two experiments. The combined HERA cross sections for inclusive NC $e^{+} p$ and $e^{-} p$ cross sections are based on a total integrated luminosity of about $1 \mathrm{fb}^{-1}$ and the results are displayed in fig. 2 and compared to predictions using the extracted HERAPDF2.0 PDF sets in next-to-next-to-leading order (NNLO) precision. The total uncertainties are below $1.5 \%$ over the $Q^{2}$ range of $3 \leq Q^{2} \leq 500 \mathrm{GeV}^{2}$ and below $3 \%$ up to $Q^{2} \simeq 3000 \mathrm{GeV}^{2}$. The combination leads to significantly reduced uncertainties compared to the single individual data points and one benefits from the different reconstrution and calibration techniques used for the initial data sets.

The combined data may be integrated over $x_{\mathrm{Bj}}$ to obtain single differential cross sections as function of $Q^{2}$. These data demonstrate beautifully the electroweak unification where the $\mathrm{NC}$ and $\mathrm{CC}$ cross sections become similar at values of $\sqrt{Q^{2}}$ close to the masses of the electroweak bosons. For the NC cross sections, differences between $\mathrm{e}+\mathrm{p}$ and e-p scattering become important at these scales and the combined data allows an extraction of the structure function $x F_{3}^{\gamma Z}$ for values of $Q^{2}>1000 \mathrm{GeV}$, as displayed in fig. 3 . 

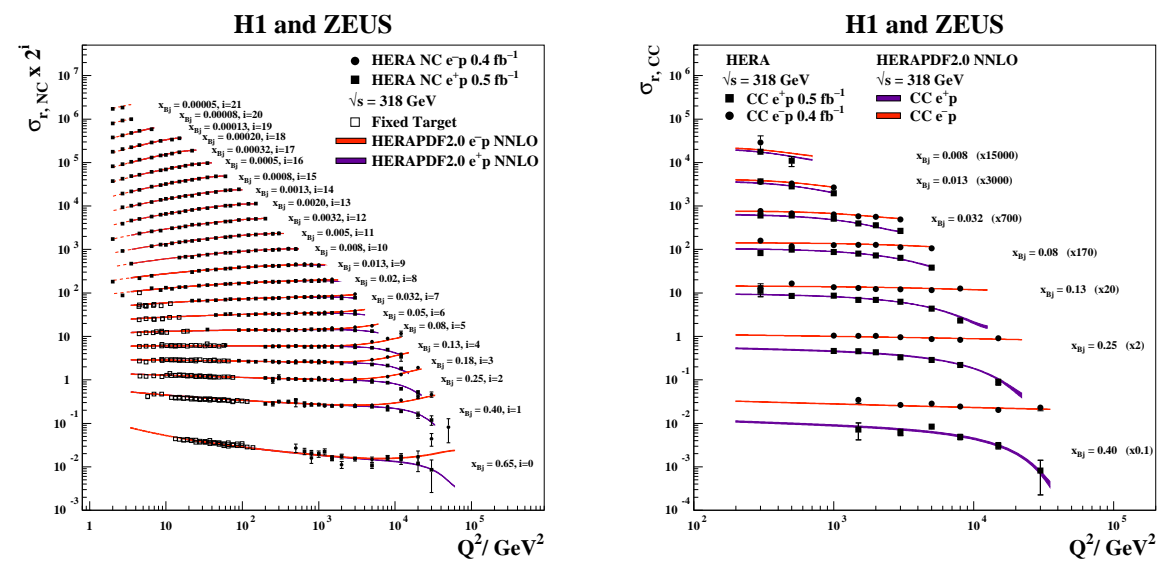

Fig. 2. The combined HERA data for the inclusive $\mathrm{NC} e^{+} p$ and $e^{-} p$ reduced cross sections together with fixed-target data and the predictions of HERAPDF2.0 NNLO (left). The bands represent the total uncertainties on the predictions. The combined HERA data for inclusive CC $e^{+} p$ and $e^{-} p$ reduced cross sections at $\sqrt{s}=318 \mathrm{GeV}$ with overlaid predictions of HERAPDF2.0 NNLO (right).
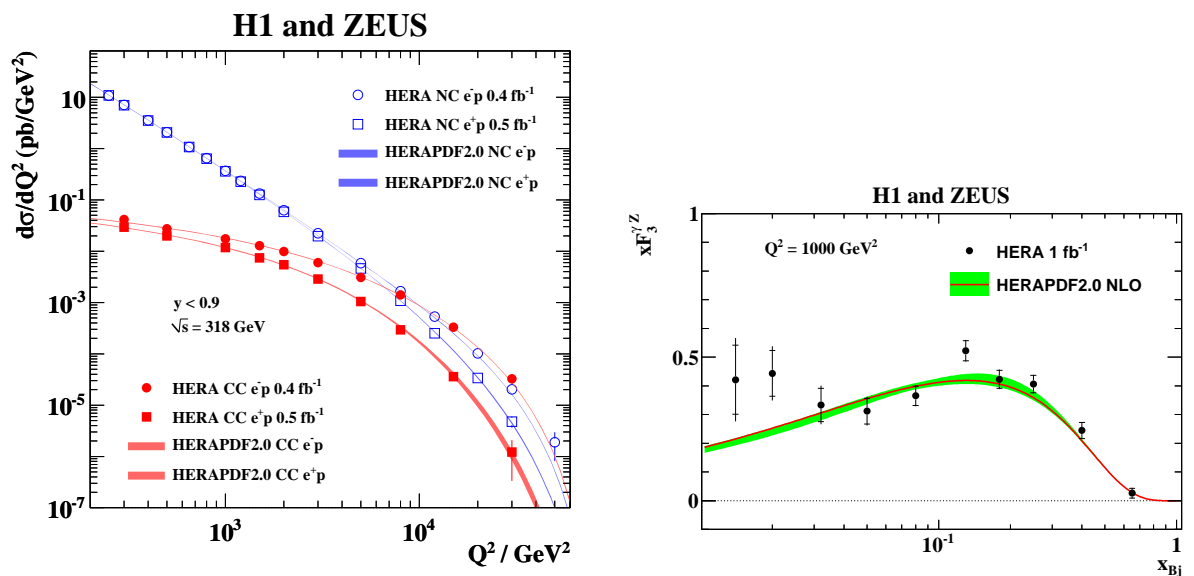

Fig. 3. The $\mathrm{NC}$ and $\mathrm{CC} e^{-} p$ and $e^{+} p$ cross sections togther with theoretical predictions using HERAPDF2.0 (left) demonstrating the electroweak unification. The extracted and predicted values of the structure function $x F_{3}^{\gamma Z}$ at $Q^{2}=1000 \mathrm{GeV}^{2}$ (right).

\subsection{HERAPDF2.0}

Within the framework of perturbative QCD, the proton is described by parton distribution functions which are interpreted as probabilities to find a parton, i.e. a gluon or quark, carrying a fraction $x$ of the proton momentum. Predictions of the perturbative QCD framework are fitted to the combined $\mathrm{NC}$ and $\mathrm{CC}$ data of 
$Q^{2} \geq 3.5 \mathrm{GeV}^{2}$, where the PDFs are parameterized at a scale $Q_{0}^{2}=1.9 \mathrm{GeV}^{2}$ and the optimal parameters to these functions are determined in that $\chi^{2}$-minimization procedure. The sets of PDFs are then functions of the factorization scale $\mu_{f}$ and $x$ and are obtained by solving the DGLAP evolution equations to any scale $\mu_{f}^{2}$. The factorization scale is commonly identified in DIS with $\mu_{f}^{2}=Q^{2}$, where the convolution with the coefficient functions is calculated. These fits were done using the programs QCDNUM and APFEL within the HERAFitter ${ }^{\mathrm{b} 3}$ framework in LO, NLO and NNLO accuracy and the resulting PDF sets are denoted HERAPDF2.0.
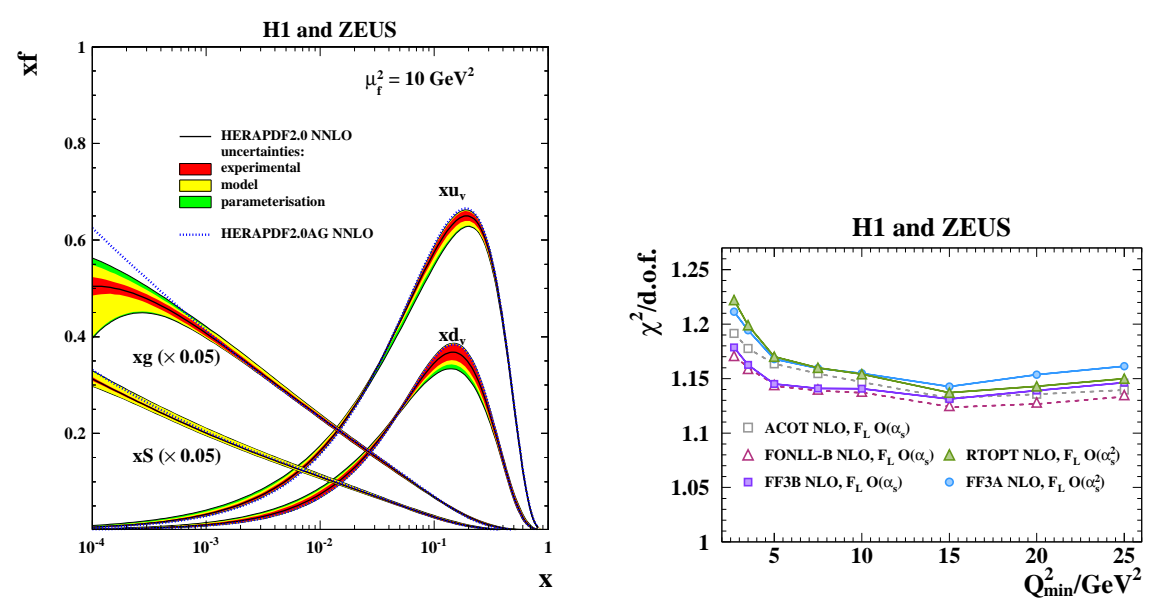

Fig. 4. The quark distribution functions $x u_{v}, x d_{v}, x S=2 x(\bar{U}+\bar{D})$ and the gluon distribution function, $x g$, of HERAPDF2.0 NNLO at $\mu_{f}^{2}=10 \mathrm{GeV}^{2}$ (left). The dotted lines represent the results obtained with an alternative paremeterization for $x g$. The dependence of $\chi^{2} / n_{\mathrm{dof}}$ on $Q_{\min }^{2}$ for HERAPDF2.0 fits using various heavy-flavour schemes at NLO and NNLO (right). The $F_{L}$ contributions are calculated using matrix elements of different orders in $\alpha_{s}$ as indicated in the legend. Due to the cut in $Q^{2}$, the number of degrees of freedom drops from 1148 at $Q_{\min }^{2}=2.7 \mathrm{GeV}^{2}$ to 868 for $Q_{\min }^{2}=25 \mathrm{GeV}^{2}$.

Fig. 4 shows a summary plot at the scale $\mu_{f}^{2}=10 \mathrm{GeV}^{2}$ of the valence quarks, total sea quarks and the gluon PDFs of the HERAPDF2.0 in NNLO. The red band indicates the total experimental uncertainty, the yellow band shows model uncertainties from the variations of the cut on the minimum value of $Q^{2}$, the values of the charm and bottom masses, and the fractional contribution of the strangePDF. Uncertainties on the variations of the parameterisations are small and shown in green.

The $\chi^{2}$ per degree of freedom, $n_{\text {dof }}$, of the HERAPDF2.0 fit is $1357 / 1131$. It was observed, that this value changes with a variation of the cut on $Q^{2}$ for NLO and

\footnotetext{
b The HERAFitter package is publicly available and is now called "xFitter". All results have been validated with the alternative program used for previous analyses.
} 
NNLO fits (compare fig. 4). The cut was further studied for various heavy quark schemes where, however, it depends mostly to the order to which $F_{L}$ is evaluated, but the raise of $\chi^{2} / n_{\text {dof }}$ at low values of $Q_{\min }^{2}$ suggests that further contributions beyond the DGLAP formalism, e.g. resummation of $\ln (1 / x)$ terms, non-linear evolution equations or non-perturbative effects, may become important.
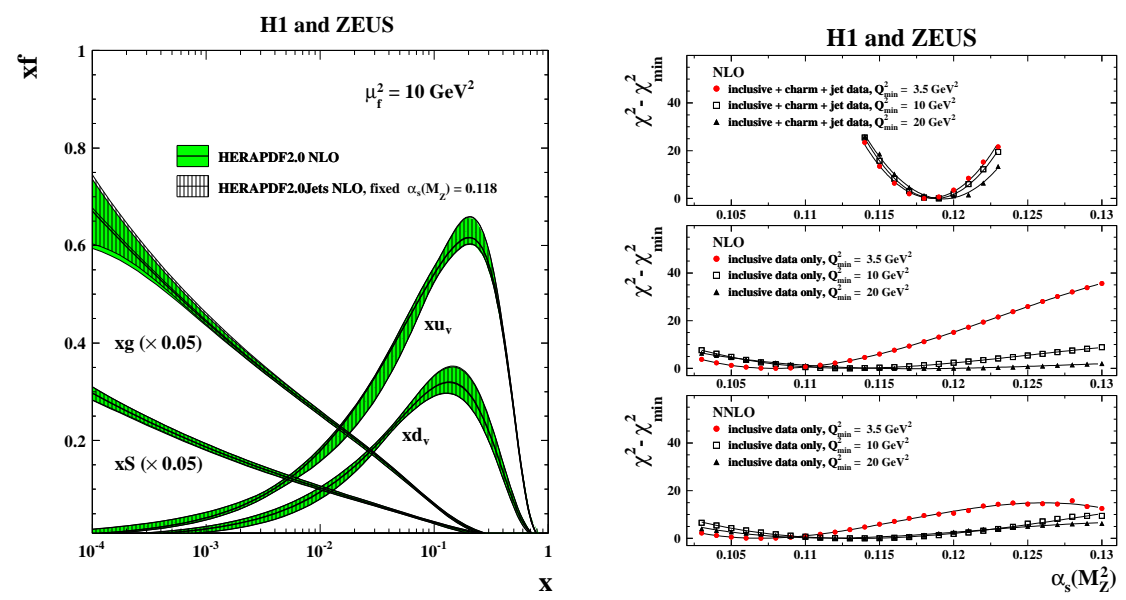

Fig. 5. Parton distribution functions of HERAPDF2.0Jets, including charm and jet data, compared to HERAPDF2.0 NLO (left). Values of $\chi^{2}-\chi_{\min }^{2}$ vs. $\alpha_{s}\left(M_{Z}\right)$ for PDF fits with three different values of $Q_{\min }^{2}$ (right). The inclusion of jet data in the QCD fit (top) constrain the value of $\alpha_{s}\left(M_{Z}\right)$ significantly better than the fits to inclusive data alone.

Furthermore multiple data sets on jet production from the H1 and ZEUS experiments as well as combined data on charm production is added to the HERAPDF2.0 PDF fit (see also sec. 3 and 4). The charm data do not change the results significantly, because their constraining power is amortized since they were already employed for the determination of the optimal charm mass parameter. The jet data are powerful to constrain the strong coupling constant as it is illustrated on the basis of the $\chi^{2}$-parabolas in fig. 5 . The extracted value from the PDF fit, if $\alpha_{s}\left(M_{Z}\right)$ is an additional free parameter, is

$$
\alpha_{s}\left(M_{Z}\right)=0.1183 \pm 0.0009_{\exp } \pm 0.0005_{\bmod / \text { param }} \pm 0.0012_{\text {had }}^{+0.0037}{ }_{-0.030 \text { scale }}^{+}
$$

The PDFs do not change visibly by including jet data, since this optimal value of $\alpha_{s}\left(M_{Z}\right)$ is already close to the default value of the fit of $\alpha_{s}\left(M_{Z}\right)=0.118$, but the jet data help to disentangle the correlation between the parameters of the gluon distribution and $\alpha_{s}\left(M_{Z}\right)$.

\subsection{Use of HERA inclusive data}

The final HERA inclusive data sets are valuable input to further studies. They were employed for an extraction of an upper limit on an effective quark radius ${ }^{4}$ 
of $R_{q}^{2}<\left(0.43 \cdot 10^{-16} \mathrm{~cm}\right)^{2}$, which improves an earlier limit based on H1 data alone $^{5}$. Furthermore, parameters of the electroweak theory may be extracted, where couplings of the quarks to the $Z$-boson may be determined with improved precision, and also the mass of the $W$-boson can be tested ${ }^{6}$.

\section{Jet production in DIS}

Measurements of jet production cross sections in NC DIS give direct access to the strong coupling constant $\alpha_{s}\left(M_{Z}\right)$, since these are performed in the Breit frame of reference, where the virtual boson collides head-on with the parton inside the proton. In order to produce a parton, which then hadronizes into a jet, with significant transverse momentum, $p_{T}^{\text {jet }}$, at least one strong vertex must be present. The H1 collaboration has measured inclusive jet, dijet and trijet cross sections ${ }^{7}$ in the $\mathrm{NC}$ DIS kinematic region of $150<Q^{2}<15000 \mathrm{GeV}^{2}$ for jets with $p_{T}^{\text {jet }}>5 \mathrm{GeV}(7 \mathrm{GeV}$ in the case of inclusive jets). Jets are found using the $k_{\mathrm{T}}$ and anti- $k_{\mathrm{T}}$ cluster algorithm. The jet cross sections are further normalized to the NC DIS cross sections in the respective $Q^{2}$ bin, which helps to eliminate normalization uncertainties. These normalized multijet cross sections are compared to NLO predictions in fig. 6, where an overall good agreement is observed.
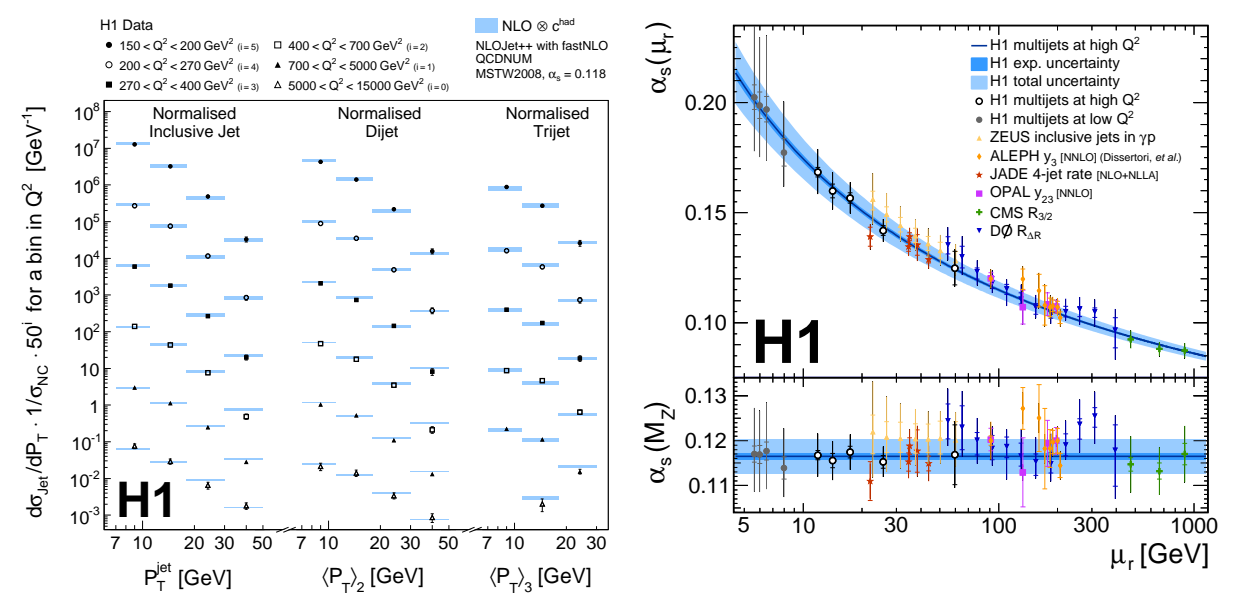

Fig. 6. Comparison of normalized inclusive jet, dijet and trijet cross sections to NLO QCD predictions as function of $Q^{2}$ and transverse momenta observables (left). Values of $\alpha_{s}\left(M_{Z}\right)$ and corresponding values of $\alpha_{s}\left(\mu_{r}\right)$ extracted from normalized multijet cross sections (open circles) compared to values extracted from other jet data (right). The prediction for the running of $\alpha_{s}\left(\mu_{r}\right)$ using the best-fit value is also shown with related uncertainies.

The high precision of the data allows for an experimentally precise extraction of the strong coupling constant of

$$
\alpha_{s}\left(M_{Z}\right)=0.1185 \pm 0.0008_{\exp } \pm 0.0038_{\text {theo }} .
$$


Fits to sets of data points at similar scales confirm the assumption of the running of the strong coupling constant, and consistency with other H1 and ZEUS jet data ${ }^{8}$ and jet data from other colliders is observed, as shown in fig. 6 .

The measurements of inclusive jet ${ }^{9}$, dijet ${ }^{10}$ and trijet ${ }^{11}$ cross sections from the ZEUS experiment in NC DIS using HERA-II, show also a good agreement with NLO predictions within the uncertainties. It is expected that improved predictions in NNLO will help to reduce the dominant theoretical uncertainty on $\alpha_{s}\left(M_{Z}\right)$ in the future.

\section{Heavy flavour production in DIS}

Due to their long lifetime or their semi-leptonic decays, charm mesons can be well measured at HERA and thus the measurements of beauty and charm production in $e p$ scattering are important processes for the studies of perturbative QCD and to determine the masses of those two heavy quarks. The presence of multiple scales in the process, i.e. the virtuality of the initiating photon $Q^{2}$, the transverse momenta of the outgoing hadron, $p_{T}$, and the heavy quark mass $m_{\mathrm{c} / \mathrm{b}}$ complicates the QCD calculations for such processes. Furthermore, the dynamic production of heavy quarks in the DGLAP formalism can be investigated.
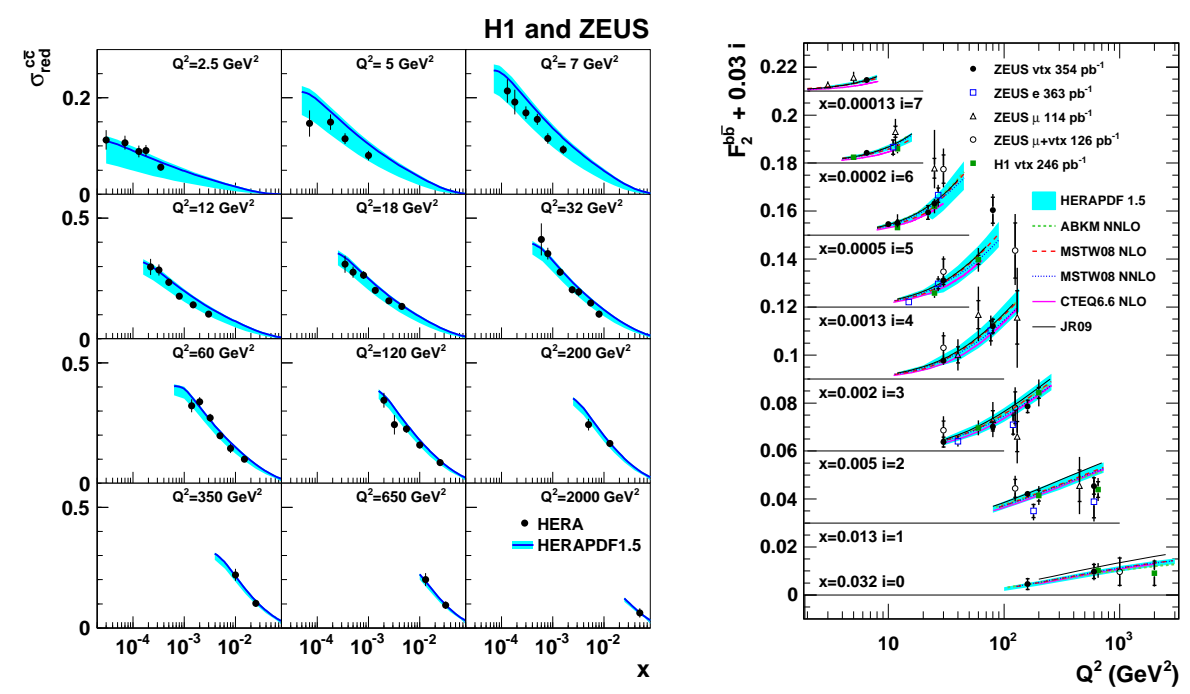

Fig. 7. Combined reduced charm-production cross sections $\sigma_{\text {red }}^{c \bar{c}}$ in NC DIS as a function of $x$ and $Q^{2}$ compared to predictions based on HERAPDF1.5 (left). The full line represents the prediction using $m_{c}=1.4 \mathrm{GeV}$ and the band indicates the full PDF uncertainty. Measurements of the beauty structure function $F_{2}^{b \bar{b}}$ in DIS from the ZEUS and H1 experiments compared to several NLO and NNLO predictions (right). The PDF uncertainty band is dominated by the variation of $m_{b}$.

A large number of data on charm production by H1 and ZEUS are combined ${ }^{12}$ 
into common cross sections in the phase space $2.5 \leq Q^{2} \leq 2000 \mathrm{GeV}^{2}$ and results are shown in fig. 7. For this combination cross sections in the restricted phase space are extrapolated to the full phase space using NLO predictions. The best description of the data is obtained by NNLO fixed-flavor-number-scheme predictions from the ABM group.

Inclusive jet production is measured by ZEUS in events containing beauty or charm quarks, exploiting the long lifetimes and large masses of $b$ and $c$ hadrons ${ }^{13}$. As example here, the extracted structure function $F_{2}^{b \bar{b}}$ from these data is compared in fig. 7 to earlier measurements by $\mathrm{H}^{14}$ and ZEUS, and consistency is found. Also the extracted values are in agreement with NLO and NNLO predictions from different heavy-quark schemes used by the PDF groups.
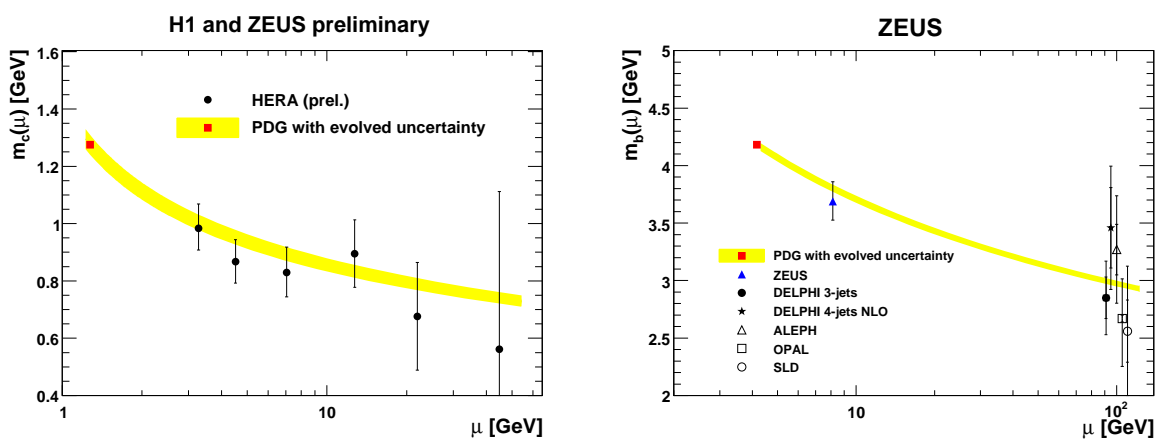

Fig. 8. Values of the charm mass $m_{c}(\mu)$ in the $\overline{\mathrm{MS}}$ running scheme as function of the scale $\mu$ (left). The values of $m_{c}(\mu)$ are obtained from translations of the fit parameters $m_{c}\left(m_{c}\right)$ and the scale $\mu$ denotes the representative scale of the used data. The beauty mass, $m_{b}(\mu)$, in the $\overline{\mathrm{MS}}$ running scheme as function of the typical scale $\mu$ of the data (right). The values $m_{b}(\mu)$ are obtained from $m_{b}\left(m_{b}\right)$ by solving the renormalization group equation as it is also illustrated by the yellow band.

The combined charm data is employed for an extraction of the charm quark mass $m_{c}$ in a QCD analysis in fixed-flavor-number-scheme at NLO using the $\overline{\mathrm{MS}}$ running mass definition and a value of

$$
m_{c}\left(m_{c}\right)=1.26 \pm 0.05_{\text {exp }} \pm 0.03_{\text {mod }} \pm 0.02_{\text {param }} \pm 0.02_{\alpha_{s}} \mathrm{GeV}
$$

is extracted ${ }^{15}$. The extraction may be repeated to subsets of data points at different scales and such the charm quark mass is probed at different scales. The six extracted values of $m_{c}\left(m_{c}\right)$ may be used to illustrate the charm quark running in the $\overline{\mathrm{MS}}$ renormalisation scheme, as displayed in fig. 8.

The running beauty-quark mass in the $\overline{\mathrm{MS}}$ scheme was determined from an NLO QCD fit in the fixed-flavour-number scheme to the reduced beauty cross sections ${ }^{16}$ to:

$$
m_{b}\left(m_{b}\right)=4.07 \pm 0.14_{\text {fit }} \stackrel{+0.01}{+0.07} \text { mod } \stackrel{+0.00}{0.00} \text { param } \stackrel{+0.08}{-0.05} \text { theo } \mathrm{GeV} .
$$


The value of $m_{b}\left(m_{b}\right)$ is compared to extractions at different scales in fig. 8 .

The extracted values of the charm and beauty quark masses agree with the world average values.

\section{QCD studies in diffractive DIS}

Diffractive hadron-hadron interactions are found to be suppressed as compared to NLO predictions which are based on diffractive-PDFs (DPDFs) obtained from HERA diffractive data ${ }^{17}$. Dijet production in diffractive photoproduciton (PHP) allows the process to be studied in a similar environment to that of two interacting hadrons and to test the model of proton vertex factorization. In diffractive DIS, dijet production measurements provide further insights to the nature of the diffractive exchange. Under the assumption of proton vertex factorization, dijet data may then provide direct access to the gluon content of the DPDFs.

The $\mathrm{H} 1$ experiment has measured differential dijet production cross sections ${ }^{18}$ in diffractive PHP $\left(Q^{2}<2 \mathrm{GeV}^{2}\right)$ as well as in diffractive DIS $\left(4<Q^{2}<80 \mathrm{GeV}^{2}\right)$ in the kinematic range of $0.01<x_{\mathbb{P}}<0.024$. The leading final state proton is tagged in the H1 Very Forward Proton Spectrometer (VFPS) $220 \mathrm{~m}$ away from the interaction point, thus providing a complementary experimental method to previous analyses in PHP and DIS. The DIS data are well described by NLO predictions using DPDFs, while the NLO predictions for the photoproduction regime overestimate the data by about a factor of two, as displayed in fig. 9, and no kinematic dependence in one of the studied observables is observed. The result confirms previous measurements from H1, and with the new data possible contributions from proton-dissociative processes alone are excluded as an explanation for the observed suppression.

In a similar DIS kinematic domain, inclusive dijet cross sections are further measured with the diffractive events identified with the large rapidity gap method ${ }^{19}$. The high statistical precision allows for double-differential cross section measurements. Good agreement with the NLO prediction is found (as displayed in fig. 9) and the data are employed for an extraction of the strong coupling constant of $\left.\alpha_{s}\left(M_{Z}\right)=0.119_{\exp } \pm 0.004\right) \exp \pm 0.012_{\mathrm{DPDF}, \text { theo }}$. This exhibits the data precision to be higher than the NLO accuracy obtained, limited by scale variations and the knowledge of the DPDFs. This result demonstrates the capability that these data may be used in future DPDF fits.

Dijet production is further studied as exclusive dijet cross sections in diffractive DIS for $Q^{2}>25 \mathrm{GeV}$ and for $\gamma^{*} p$ center-of-mass energies of $90<W<250 \mathrm{GeV}$ by the ZEUS experiment ${ }^{20}$. The study of of the angle $\phi$ between the $\gamma^{*}$-dijet plane and the $\gamma^{*} e^{ \pm}$plane in the rest frame of the diffractive final state, reveals that the Two-Gluon-Exchange model is more successful in describing the data than the Resolved-Pomeron model (compare fig. 10).

Further insights into the nature of the diffractive processes is gained by studying prompt photons in diffractive photoproduction ${ }^{21}$, since photons do not participate in the color reconnection and hadronisation processes and thus give direct access to 

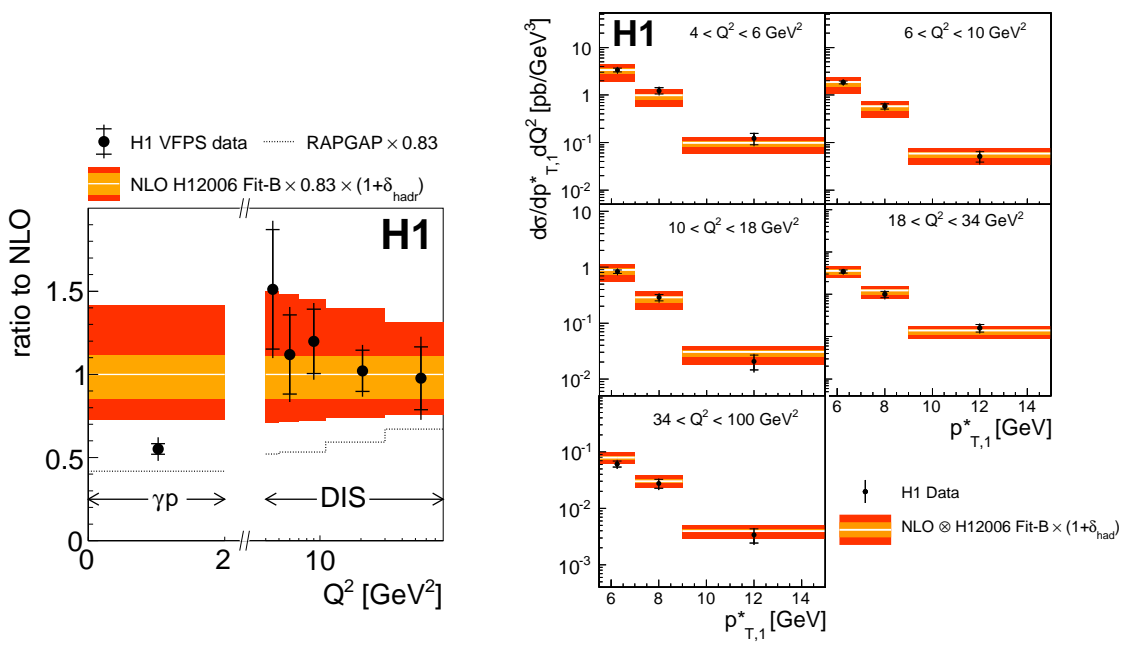

Fig. 9. Dijet production in diffractive DIS and photoproduction compared to prediction at NLO assuming proton-vertex factorization and DPDFs from H1 (left), where the leading final state is identified in the VFPS. The photoproduction cross sections are found to be overestimated by the NLO predictions. Dijet production in diffractive DIS compared to NLO predictions (right), where diffractive events are identified with the large rapidity gap method.
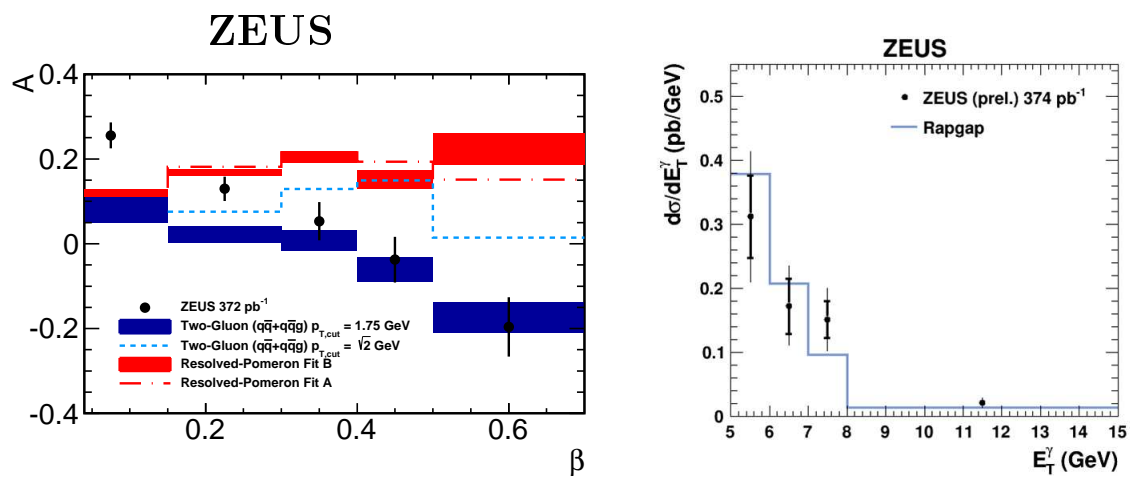

Fig. 10. The shape parameter $A$, derived from the angle $\phi$, for exclusive dijet production in diffractive DIS compared to predictions obtained using the Two-Gluon-Exchange model and the Resolved-Pomeron model (left). Differential cross sections for isolated photons accompanied by a jet in diffractive PHP as functions of photon transverse-energy, $E_{\mathrm{T}}^{\gamma}$, compared to normalized prediction from Rapgap (right).

the hard process itself. The studies of photon cross sections in association with a jet by the ZEUS experiment show good agreement of data and Monte Carlo predictions from Rapgap in the region where the DPDFs have been constrained by data (see fig. 10). 
12 Daniel Britzger

\section{Conclusions}

Almost ten years after ending of data taking at the HERA accelerator the H1 and ZEUS experiments are finalizing their physics analyses. The measurements of the large variety of processes in $e p$ scattering provide unique opportunities for studies of the phenomenolgy of QCD and the determination of its fundamental parameters. Here we focussed on more recent results from the HERA experiments, while there is a large variety of earlier results worth to mention, but which is beyond the scope of this article.

\section{References}

1. H. Abramowicz et al. [H1 and ZEUS Collaborations], Eur. Phys. J. C 75 (2015) 12, 580

2. https://wiki-zeuthen.desy.de/HERAverager

3. S. Alekhin et al. [HERAFitter Developer Team], Eur. Phys. J. C 75 (2015) 7, 304

4. H. Abramowicz et al. [ZEUS Collaboration], Phys. Lett. B 757 (2016) 468

5. F.D. Aaron et al. [H1 Collaboration], Phys.Lett. B 705 (2011) 52

6. H. Abramowicz et al. [ZEUS Collaboration], Phys. Rev. D 93 (2016) 092002

7. V. Andreev et al.[H1 Collaboration], Eur. Phys. J. C 75 (2015) 2, 65

8. H. Abramowicz et al. [ZEUS Collaboration], Nucl. Phys. B 864 (2012), 1

9. ZEUS Collaboration, ZEUS preliminary, ZEUS-prel-10-002 (2010)

10. H. Abramowicz et al. [ZEUS Collaboration], Eur. Phys. J. C 70 (2010) 965

11. ZEUS Collaboration, ZEUS preliminary, ZEUS-prel-14-008 (2014)

12. H. Abramowicz et al. [H1 and ZEUS Collaborations], Eur. Phys. J. C 73 (2013) 2, 2311

13. H. Abramowicz et al. [ZEUS Collaboration], JHEP 1409 (2014) 127

14. F.D. Aaron et al. [H1 Collaboration], Eur. Phys. J. C 65 (2010) 89

15. H1 and ZEUS Collaborations, H1 and ZEUS preliminary, H1prelim-14-071 and ZEUSprel-14-006 (2014)

16. O. Behnke et al., Prog. Part. Nucl. Phys. 84 (2015) 1

17. A. Aktas et al. [H1 Collaboration], JHEP 0710 (2007) 042

18. V. Andreev et al. [H1 Collaboration], JHEP 1505 (2015) 056

19. V. Andreev et al. [H1 Collaboration], JHEP 1503 (2015) 092

20. H. Abramowicz et al. [ZEUS Collaboration], Eur. Phys. J. C 72 (2012) 1869

21. ZEUS Collaboration, ZEUS preliminary, ZEUS-prel-15-001 (2015) 